\title{
ANÁLISIS DE LA IMPLICACIÓN Y LA REGULACIÓN DEL TRABAJO DEL ALUMNO MEDIANTE EL USO DE HERRAMIENTAS VIRTUALES
}

\author{
David Hortigüela Alcalá1 Universidad de Burgos. España. \\ davidhortiguela@ubu.es
}

\author{
Ángel Pérez Pueyo. Universidad de León. España \\ angel.perez.pueyo@unileon.es
}

\section{RESUMEN}

Esta investigación se ha llevado a cabo a lo largo del primer semestre del curso 20142015 en la Facultad de Educación de Burgos. Se analizan las variables de implicación y regulación del trabajo del alumno a partir de la elaboración de vídeos en directo con Google Hangout y su subida a Youtube. Los estudiantes que participan en la investigación son 126, todos ellos pertenecientes a una asignatura del Grado en Primaria. El grupo A ha elaborado los vídeos como instrumento de aprendizaje mientras que el grupo B no ha utilizado herramientas virtuales. Se realiza un pretestpostest, analizando el grado de modificación de las variables estudiadas al finalizar la asignatura. Se utiliza un método de investigación mixto, tanto cuantitativo (descriptivo e inferencial) como cualitativo (entrevistas). El grupo que ha utilizado los vídeos (A) presenta una mayor regulación de trabajo, tanto en relación al pretest, como en relación al grupo B. En el grupo A ha sido la asiduidad en el manejo de las redes sociales la variable sobre la que se han encontrado diferencias significativas en relación a la aplicabilidad del aprendizaje obtenido. Las entrevistas realizadas a los docentes reflejan un nivel muy dispar en el conocimiento y manejo de las herramientas virtuales con fines formativos.

\section{PALABRAS CLAVE}

Herramientas virtuales - Implicación hacia el trabajo - Motivación - Regulación del trabajo - Reflexión sobre el aprendizaje - Percepción docente - Percepción del alumno - Método mixto - Estrategias metodológicas

\footnotetext{
${ }^{1}$ David Hortigüela Alcalá: Doctor Internacional en Educación. Máster en Educación y Sociedades Inclusivas. Licenciado en Ciencias de la Actividad Física y del Deporte. Maestro Especialista en Educación Física. davidhortiguela@ubu.es
} 


\title{
ANALYSIS OF THE INVOLVEMENT AND REGULATION OF STUDENT WORK USING VIRTUAL TOOLS
}

\begin{abstract}
This research was carried out during the first semester of 2014-2015 at the Faculty of Education of Burgos. Variables involvement and regulation of student work are analyzed from the development of live videos with Google Hangout and upload to Youtube. Students participating in the research are 126, all belonging to a subject of Degree in Primary. Group A has produced the videos as a learning while group B did not use virtual tools. A pretest-posttest is done by analyzing the degree of modification of the variables at the end of the course. Mixed method research, both quantitative (descriptive and inferential) and qualitative (interviews) is used. The group has used the video (A) has a greater regulation of work, both in relation to the pretest, as compared to group B. In group A has been frequently in the management of the variable social networks on which found significant differences in relation to reflection on the learning generated. Interviews with teachers reflect a very different level in the understanding and management of virtual tools for training purposes.
\end{abstract}

\section{KEY WORDS}

Virtual tools - Implication to work - Motivation - Regulation of work - Reflection on learning - teaching Perception - Perception student - Mixed method - Methodological strategies

\section{INTRODUCCIÓN}

Es incuestionable el incremento exponencial que se ha producido en la última década en relación a la utilización de herramientas virtuales y el acceso a la información de manera digital (Marín, 2014). Esta situación también acontece en el ámbito educativo, donde son infinidad las opciones existentes para profundizar y compartir la información. Por lo tanto, y tal y como establecen Liu, Liu \& Chi (2014) ya no es tan importante la transmisión de los contenidos al alumno por parte del docente como la regulación del trabajo y la búsqueda de metodologías que favorezcan la motivación y la implicación del estudiante sobre aquello que aprende. La información está presente, es más, existe una saturación de la misma, por lo que se convierte en relevante la delimitación de estrategias que hagan que el alumno sea un miembro activo del proceso y no un mero receptor de información (Detlor, Booker, Serenko \& Julien, 2012).

Son multitud las experiencias educativas en diversas etapas que presentan la utilización de plataformas virtuales y distintas herramientas asociadas para estructurar los procesos formativos (p.e. Blanco, 2013; Flores, 2012; Roith, 2013). Una de las variables que regulan su uso, además de la complejidad y el volumen de la información manejada, es la autonomía que ha de tener el alumno en su manejo, 
alcanzando en el ámbito universitario los mayores niveles. Como indican Lau and Lee (2015) no se trata de evaluar el dominio que tenga el estudiante sobre una herramienta concreta, sino de reflexionar sobre el uso formativo que derive de la misma y la aplicabilidad y relación que tenga con los contenidos abordados en el aula.

Las posibilidades existentes para incorporar las herramientas virtuales con carácter formativo en el aula son infinitas, pero necesitan una adecuación y adaptación a la tipología de la asignatura, las características de los alumnos y los objetivos que se quieren conseguir (Vargas-Vargas, Mondejar-Jimenez, Santamaria, Alfaro-Navarro, Fernandez-Aviles, 2011). Una herramienta virtual en sí misma no es positiva ni negativa, ya que su potencial deriva del uso de la misma (Armstrong, 2011). Lo que es evidente, es que en la actualidad es fundamental plantear recursos evaluativos en las clases que favorezcan la reflexión del alumno sobre aquello que se aprende, permitiendo profundizar en el aprendizaje, contrastar datos con otros miembros de la comunidad educativa y compartir experiencias con compañeros de clase más allá de las horas presenciales. Una de las posibilidades para poder incorporar las herramientas virtuales es el trabajo en el aula mediante grupos, utilizando técnicas estructuradas bajo el aprendizaje cooperativo. Para ello es fundamental delimitar claramente desde el comienzo los objetivos que tiene que conseguir el grupo, los roles que va a desempeñar cada uno de sus integrantes y el modo en el que van a regular su trabajo (Cifuentes \& Meseguer, 2015). El ejemplo abordado en esta investigación cumple estos requisitos, articulándose a partir de la elaboración de vídeos grupales, que posteriormente son subidos a la red, y en los que se profundiza sobre los contenidos trabajados en clase.

En este sentido, la búsqueda de información en vídeos (sean tutoriales, divulgativos, de recapitulación de ideas...) es un recurso común y habitual entre el alumnado, muchas veces realizado de manera poco estructurada y sin un seguimiento formativo por parte del profesorado (Craig \& Friehs, 2013). Sin embargo, pueden suponer una fuente de conocimiento de verdadera aplicación para ampliar los recursos trabajados y contrastar información. Además implica una autonomía del alumno en la búsqueda y su propia autogestión de recursos, lo que puede repercutir en una mayor motivación hacia aquello que se aprende. Por lo tanto, y si somos conocedores de los beneficios que pueden conllevar este tipo de procedimientos... ¿por qué no permitir que el alumno cree sus propios videos con el fin de que sean elemento de reflexión por toda la clase? Es a partir de esta pregunta de dónde nace la presente investigación.

Como establece Martínez-Berruezo and García-Varela (2013) no cabe ninguna duda de que uno de los principales fines que persigue el sistema educativo actualmente es que el alumno se motive hacia el aprendizaje, y para ello necesitamos establecer metodologías de trabajo que favorezcan que el alumno se implique en las tareas sin necesidad de que únicamente se hagan por obligatoriedad. Algunas investigaciones en esta línea como la de Kenny and Fluck (2014) indican que uno de los mayores problemas en el ámbito universitario se encuentra en la falta de costumbre que tiene el alumno a la hora de regular su trabajo, viéndose muchas veces desbordado al final de curso por su mala gestión del tiempo. Esto se une a otros factores como el escaso 
tiempo de docencia del que se dispone en las asignaturas y la utilización de metodologías articuladas a partir de la lección magistral y que no favorecen la evaluación formativa. Es por ello que cuando el alumno utiliza y elabora sus propios recursos (como en el caso del vídeo) de manera autónoma y se delimitan instrumentos grupales en los que se registra el trabajo desarrollado, el aprendizaje generado es más significativo y transferible a otros contextos (Del Valle, Morales \& Sumano, 2011).

Este tipo de metodologías abiertas y participativas han de regirse por la implementación de un feedback entre profesor y alumno, tanto en el consenso previo de la metodología y los instrumentos a utilizar como en el sistema de evaluación empleado. Del mismo modo es fundamental que los alumnos emitan juicios de valor a sus compañeros sobre un trabajo realizado de manera conjunta, lo que convierte a las coevaluaciones, sean estas de carácter intra o intergrupal, como un procedimiento idóneo para involucrar al alumno en su proceso de enseñanza (Rodríguez, Ibarra \& Jiménez-Vergara, 2013). Este tipo de vías evaluativas puede implantarse a lo largo de la asignatura, siempre con carácter regulado y con el fin fundamental de profundizar en los ejes temáticos abordados.

Queda por lo tanto reflejado la relevancia que tiene la metodología empleada por el docente, así como su propio rol en la asignatura, para generar procesos de aprendizaje dialógicos, constructivos y satisfactorios. En relación a este último aspecto, estudios como el de Bulman, Lathlean and Gobbi (2014) indican que uno de los indicadores que más incide en la reflexión sobre la práctica docente es la percepción que tiene el alumno sobre el aprendizaje recibido. En este sentido, aunar puntos de vista y compartir vivencias sobre los procedimientos empleados a lo largo de la asignatura supone entender el proceso de enseñanza de manera igualitaria, encontrando las fortalezas y debilidades que inherentemente quedan implícitas cuando se enseña algo a alguien. Como establecen Gamlem and Smith (2013), cuando no escuchamos lo que el alumno piensa sobre lo que pretendemos enseñar estamos cayendo en uno de los principales errores que sustentan el principio educativo, que es enfocar el proceso de enseñanza bajo un enfoque unidireccional y no democrático. Por lo tanto, y atendiendo a estos enfoques evaluativos centrados en la percepción del alumno y el docente sobre los procesos de enseñanza generados, en el presente estudio se analiza en qué medida la elaboración de videos como herramienta de aprendizaje favorece la implicación y la regulación del estudiante hacia el trabajo.

\section{OBJETIVOS}

Analizar la valoración del estudiante en relación a la implicación y la regulación del trabajo antes y después de vivenciar la experiencia desarrollada con la elaboración y exposición de vídeos.

Examinar las diferencias que se establecen entre grupos respecto a la transferencia en el aprendizaje obtenido en relación a las variables independientes de edad, uso de redes sociales y experiencia previa en el Hangout.

Comprobar las percepciones de los docentes sobre la conveniencia de utilizar herramientas virtuales en el aula como elemento motivador y de aprendizaje. 


\section{METODOLOGÍA}

\subsection{Participantes}

Son 126 alumnos los que han participado en el presente estudio, dividiéndose en $58.7 \%$ mujeres y $41.3 \%$ hombres. La media de edad de los alumnos es de 22.21 años $(\mathrm{DT}=3.69)$. La materia en la que se ha llevado a cabo la experiencia es Didáctica de la Educación Física, asignatura obligatoria de segundo curso del Grado en Primaria. Se generan dos grupos, A de mañana, constituido por 67 alumnos, y B, de tarde, de 59. En grupo A los alumnos han elaborado sus propios vídeos con la herramienta Hangout para utilizarlo como recurso formativo, mientras que en el grupo B no se ha empleado ninguna herramienta virtual como instrumento de aprendizaje. Cada grupo ha sido impartido por un docente diferente, uno con cinco años de experiencia en el ámbito universitario (grupo A) y otro con 35 (grupo B). La muestra responde a parámetros de normalidad, obteniendo una $p=.214$ en la prueba de Shapiro-Wilk.

\subsection{Instrumentos}

\subsubsection{Cuantitativos}

Dentro del análisis cuantitativo se emplea el cuestionario elaborado por Castejón, Santos y Palacios (2013) que versa sobre la metodología y evaluación en la formación inicial del profesorado. Se comprueba la aplicabilidad y utilidad del instrumento, ya que además de correlacionar positivamente cada uno de los ítems en relación al ítem total se realizó una validación por un grupo de expertos en la temática a través de un proceso formativo de retroalimentación hasta alcanzar un consenso. La fiabilidad del instrumento es elevada, habiendo obtenido un alfa de Cronbach de 0.834, aceptado como fiable (Corbetta, 2007). Cada una de las cuestiones se construyó a partir de la identificación de las variables predictivas, adaptadas estas a los objetivos del estudio. Se aplica un nivel de confianza del $95 \%$.

Son 17 ítems los que componen el cuestionario, estructurando las respuestas de manera escalonada, donde 1 es nada y donde 5 es Mucho. Los índices obtenidos en la matriz de covarianzas, presentaron ajustes satisfactorios para el índice RMSEA (Root Mean Square Error Aproximation) $=0.072$. En este índice los valores inferiores a .05 indican un buen ajuste, y valores de hasta .08 representan errores razonables de aproximación. En el CFI (Comparative Fit Index) se alcanza un valor de 0.9, indicativo de buen ajuste.Tras llevarse a cabo el análisis factorial en el estudio, son dos factores de trabajo los resultantes:

1- Implicación hacia las tareas (nueve ítems): se integran cuestiones relacionadas con la motivación hacia las tareas, utilidad del trabajo realizado, seguimiento llevado a cabo y percepción positiva sobre su realización.

2- Regulación de la carga de trabajo (ocho ítems): se abordan aspectos vinculados con la planificación del trabajo, el reparto de roles, el registro de la información y la retroalimentación establecida con el docente a lo largo de la asignatura.

\subsubsection{Cualitativos}


Se utilizó una entrevista semi-estructurada a los dos docentes que impartieron la asignatura, uno en el grupo A y otro en el grupo B. El principal fin era conocer en profundidad cuál era la valoración que tenían los profesores acerca de la utilización de instrumentos asociados a las NNNT, analizado su experiencia interna y su aplicación metodológica (Schatz, 2012). Se desarrolló una estructura de preguntas relacionadas con los factores de estudio y con los medios de trabajo utilizados en las asignaturas. El hecho de que la entrevista no fuera cerrada, permitió que se realizaran otras cuestiones relacionadas, favoreciendo así una continuidad en las respuestas de los docentes. De este modo se consigue crear un diálogo pautado a modo de conversación (Schatz, 2012), indagando y profundizando en la obtención de respuestas más concretas y aplicables (Smith \& Osborn, 2003). En relación a los objetivos de la investigación y a los factores de análisis generados, se preguntaron a los docentes cinco cuestiones: 1- ¿Cómo consideras que el alumno puede implicarse hacia el proceso de aprendizaje de manera activa?, 2- ¿Crees que es importante que el alumno regule su trabajo?, 3- ¿Qué instrumentos de evaluación consideras importantes utilizar para conseguir una mayor motivación hacia la asignaturas?, 4¿En qué medida consideras favorable el uso de las NNTT para profundizar en el aprendizaje? 5- ¿Enseñas a utilizar instrumentos concretos o lo hacen de manera autónoma? ¿Por qué?

En función del tipo de contenidos abordados, los factores de análisis y la saturación obtenida en las respuestas se han generado tres categorías en las que se recapitula la información: 1- "Estrategias metodológicas utilizadas para fomentar implicación del alumno", 2- "Procedimientos e instrumentos aplicados al alumnado que favorezcan la regulación del trabajo", 3- "Enseñanza del uso de herramientas virtuales como medio para guiar y profundizar en el aprendizaje"

\subsection{Diseño y procedimiento}

La asignatura, de segundo curso, se ha impartido a lo largo del segundo semestre del año académico 2014-2015. Los participantes de los dos grupos cumplimentaron el cuestionario de manera individual antes y después de desarrollarse la asignatura. Se garantizó el anonimato y la confidencialidad en el tratamiento de los datos. Así mismo, se destacó la importancia de que contestaran con sinceridad, ya que las respuestas no influirían en nada en sus calificaciones.

Es una asignatura con dos grupos, por lo que los objetivos y las competencias a conseguir son los mismos, quedando delimitados en la guía docente. No obstante es preciso destacar cuál ha sido la metodología empleada y el modo de proceder en cada uno de los grupos: 
- Grupo A elaboración de vídeos: a partir de los contenidos abordados en la asignatura y de los aspectos reflexivos generados en las sesiones prácticas, los estudiantes realizan sus propios vídeos de trabajo. En estos vídeos los alumnos profundizan en los ejes temáticos trabajados en el aula, ampliando la información y compaginándola con la publicada por autores de referencia en la materia. Estos vídeos son subidos posteriormente a Youtube, creando un canal para toda la clase que posteriormente permite el visionado y la reflexión conjunta por la totalidad de los estudiantes. Se lleva a cabo una metodología participativa, utilizando una evaluación formativa asociada a la indagación y búsqueda de soluciones por parte del discente.

- Grupo B no elaboración de vídeos: en este grupo no se utilizan instrumentos virtuales asociados a la regulación del trabajo y la autonomía por parte del alumno. La mayor parte de las sesiones son impartidas bajo la clase magistral, compartiendo este modelo con la elaboración de sesiones por grupos. No existe una continuación y prolongación de los contenidos abordados en el aula fuera de la misma, lo que no deriva en una regulación del trabajo por el alumno, ya sea individualmente o en grupos. La única retroalimentación existente es la que realiza la profesora en el aula, no trabajándose ni la autoevaluación ni las coevaluaciones.

\subsection{Análisis empleado}

El método utilizado es el mixto, también conocido como tercer paradigma. Se han combinado técnicas cuantitativas mediante análisis descriptivos e inferenciales y cualitativa a través de entrevistas a los docentes al finalizar el proceso. El análisis se lleva a cabo tanto antes del comienzo de la asignatura como después, analizando el nivel de implicación en las tareas y la regulación del trabajo que han experimentado en cada uno de los dos grupos. Este tipo de procedimiento metodológico favorece el contraste de datos y la complementariedad de los resultados obtenidos, derivando en una mayor comprensión del porqué de los resultados. En el caso de intervenciones educativas como esta, Hall \& Ryan (2011) indican que los métodos mixtos sirven para que el profesor reflexione sobre su práctica, obteniendo una gran información sobre el nivel de aprendizaje que generan las propuestas desarrolladas.

\subsubsection{Cuantitativo}

Se lleva a cabo un tratamiento descriptivo, (medias y DT) y uno inferencial (ANOVA) para cada uno de los dos grupos. El tratamiento de los datos en el pretest-postes se hace a través de los factores de análisis, viendo si existen diferencias significativas en los factores entre grupos antes y después de haberse llevado a cabo la asignatura. En 
el ANOVA se observa si existen diferencias intragrupales para las tres variables independientes utilizadas.

\subsubsection{Cualitativo}

Se obtienen datos a partir de la entrevista realizada a los docentes, contrastando las respuestas mediante el análisis del contenido extraído en relación a las categorías generadas (Fugard \& Potts, 2015). Además se triangulan los mismos, delimitando las respuestas que tienen relación con la obtenida en los cuestionarios (Denzin y Lincoln, 1994).

El análisis del contenido se centró en la búsqueda de patrones en el texto, codificando los extractos coincidentes con los patrones cruzados (Saldaña, 2009). Se realizó una primera revisión independiente por los investigadores. Se asegura la confiabilidad, credibilidad y transferibilidad de los datos, ya que las categorías elaboradas fueron depuradas y perfeccionadas con el fin de que dieran respuesta a aquello que se quería medir (Bryman, Becker \& Sempik, 2008). Como recomiendan Beckett y Clegg (2007) se presentan extractos de texto con el fin de mostrar literalmente algunas de las respuestas que más consonancia tienen con las categorías generadas. Éstas, se han obtenido por la saturación de las respuestas, utilizando para ello el programa de registro, análisis y computación de la información WEFT QDA.

Para facilitar la exposición del texto y la comprensión de la misma por parte del lector se ha delimitado una acrónimo para cada docente, DTV (docente trabajo con vídeos) y DNV (docente no vídeos)

\section{RESULTADOS}

\subsection{Análisis cuantitativo: descriptivo}

\begin{tabular}{lcccccccc}
\hline & N & Media & DT & Var. & Media & DT & Var. \\
\hline & \multicolumn{7}{c}{ Grupo elaboración vídeos (a) } \\
\hline $\begin{array}{l}\text { F.1. Implicación en } \\
\text { tareas F.1. }\end{array}$ & 67 & 3.21 & .256 & .065 & 4.11 & .238 & .056 \\
$\begin{array}{l}\text { F.2. Regulación del } \\
\text { trabajo }\end{array}$ & 67 & 3.35 & .311 & .096 & $4.42^{\text {aa }}$ & .311 & .096 \\
\hline
\end{tabular}

\begin{tabular}{llllllll}
\hline \multicolumn{7}{c}{ Grupo no elaboración vídeos (b) } \\
\hline $\begin{array}{l}\text { F.1. Implicación en } \\
\text { tareas }\end{array}$ & 59 & 3.34 & .314 & .098 & 3.51 & .134 & .017
\end{tabular}

F.2. Regulación del

$\begin{array}{llll}59 & 3.21 & .375 & .140\end{array}$

$3.26^{\text {ba }}$

$.141 \quad .019$ 
Tabla 1. Comparación de medias por factores para cada uno de los grupos en el pretestpostest (nivel de significación en las diferencias: ${ }^{*} p<.05$ ). Nota: Los superíndices reflejan los grupos entre los que se encuentran las diferencias significativas a nivel .05

Antes de llevarse a cabo la asignatura las medias de los dos factores eran muy similares entre los dos grupos, no alcanzándose en ningún caso los tres puntos y medio. Al finalizar la asignatura los datos varían sustancialmente, obteniéndose un aumento significativo en el factor de regulación de trabajo en el grupo que elaboró los vídeos virtuales en relación al pretest. En este factor también se alcanzan diferencias significativas entre grupos, con algo más de un punto. Las medias de los dos factores son más elevadas en el postest en el grupo A, sin embargo en el grupo B también aumentan aunque ligeramente.

\subsection{Análisis inferencial: ANOVA}

En función de los factores de análisis del estudio y atendiendo a los ítems que más se relacionan con la transferencia del aprendizaje obtenido a otros contextos, se ha generado la variable cuantitativa denominada "aplicabilidad del aprendizaje". Para ello se realiza un ANOVA de una vía para grupos independientes con el fin de comprobar si existen diferencias estadísticamente entre las variables independientes de edad, asiduidad en el uso de las redes sociales y el uso previo del Hangout. Además se realiza un post hoc indicando en qué grupos se encuentra esas diferencias. La variable de edad se categoriza en: 1- "entre 20 y 22", 2- "entre 23 y 25" y 3- "más de 25". La de asiduidad en el uso de las redes sociales en: 1- "diariamente", 2- "semanalmente", 3- "nunca". La de conocimiento de la herramienta del hangout en: 1- "utilización regular", 2- "lo conozco pero no suelo utilizarlo" y 3- "no lo conozco" (tabla 2).

\begin{tabular}{cccc}
\hline APLICABILIDAD DE LO APRENDIDO & $F$ & $g l$ & $p$ \\
\hline Grupo elaboración vídeos (a) & & & \\
Edad & 87.31 & 3 & .241 \\
\hline Asiduidad uso de las redes sociales & 83.63 & 2 & $.031^{*}$ \\
\hline Uso previo del Hangout & 97.32 & 1 & .243 \\
\hline Grupo no elaboración vídeos (b) & & & \\
\hline Edad & 91.56 & 1 & .271 \\
\hline Asiduidad uso de las redes sociales & 86.12 & 2 & .172 \\
\hline Uso previo del Hangout & 78.63 & 3 & .027 \\
\hline
\end{tabular}


Tabla 2. Resumen de Anova (Bonferroni) para cada una de las variables independientes analizadas en el postest (edad, asiduidad en uso de redes sociales y conocimiento de la herramienta Hangout)

$$
\text { *p }<.05 \text { entre "diariamente" (media 4,23) y "nunca" (media 3,21) }
$$

En la única variable independiente en la que se obtienen diferencias significativas en la aplicabilidad de lo aprendido es en la de asiduidad en el uso de las redes sociales $\left(F_{(67)}=83.63, p=.031\right)$. Son, dentro del grupo que elaboró los vídeos, los que las utilizan diariamente los que encuentran una mayor transferencia en el aprendizaje obtenido en la asignatura. En el grupo B no se encuentran diferencias en ninguna de las tres variables.

\subsection{Análisis cualitativo}

Se generó una gran cantidad de información de la totalidad de respuestas establecidas por cada uno de los dos docentes, discriminando aquella que no tuviera relación con el objetivo de estudio. Se especifican extractos concretos de texto integrados en cada una de las tres categorías generadas: 1- "Estrategias metodológicas utilizadas para fomentar implicación del alumno", 2- "Procedimientos e instrumentos aplicados al alumnado que favorezcan la regulación del trabajo", 3"Enseñanza del uso de herramientas virtuales como medio para guiar y profundizar en el aprendizaje".

Estrategias metodológicas utilizadas para fomentar implicación del alumno: los dos docentes consideran necesario otorgar una practicidad a la asignatura para que los alumnos desarrollen su autonomía. Sin embargo el docente que utilizó los vídeos como instrumento de regulación del trabajo es más analítico y riguroso en los métodos empleados, destacando el compromiso que el alumnado a de adquirir en su uso.

"Los chicos tienen que vivenciar mucho las prácticas [...]". "Yo desarrollo una serie de sesiones y el resto ya las hacen ellos, creo que es la mejor manera para que se pongan en el rol de futuros docentes" (DNV). "Soy partidario de que para que el alumno se implique en las tareas hay que establecer una metodología que favorezca su motivación, comprobando que lo que hace tiene una utilidad y transferencia [...]" "No se trata tanto del qué hacer sino del para qué [...]" (DTV).

Procedimientos e instrumentos aplicados al alumnado que favorezcan la regulación del trabajo: el docente que utiliza los vídeos destaca la delimitación de criterios previos de evaluación y de calificación que garanticen la responsabilidad del alumno en la asignatura. El docente que no utilizó los vídeos virtuales no otorga tanta importancia a la delimitación de procedimientos e instrumentos concretos de trabajo:

"Siempre intento que los alumnos reflexionen sobre la práctica, regulen muy bien las tareas demandadas y sean conscientes de la importancia que tiene el trabajo en grupo con delimitación de roles, autoevaluación consensuada y coevaluaciones a los demás" (DTV) "Se 
supone que tienen que ser maduros para saber administrar su tiempo [...]" "Saben que si vienen a clase regularmente y hacen sus sesiones no tienen problemas para aprobar" (DNV).

Enseñanza del uso de herramientas virtuales como medio para guiar y profundizar en el aprendizaje: en esta categoría las diferencias entre docentes son sustanciales, ya que el docente del grupo A considera fundamental el uso de estos instrumentos para profundizar el trabajo desarrollado fuera del aula, mientras que la del grupo B no utiliza ningún instrumento virtual en su asignatura a pesar de que reconozca sus ventajas:

"No dudo de que haya un montón de posibilidades para trabajar en internet con los alumnos, pero sinceramente, si no las sé utilizar yo no puedo meterme en ese mundo [...]" "Además estoy contenta con las cosas que hago (DNV)" "El uso de los vídeos ha permitido a los chicos profundizar en aspectos trabajados en clase. Ellos deciden las características del vídeo, el material a presentar, el argumento a seguir, pero siempre siguiendo un guion delimitado [...]" "Me ha demostrado ser una herramienta que ha implicado al alumno hacia las tareas y ha servido para hacer reflexiones más profundas (DTV)

\section{DISCUSIÓN}

Se ha observado cómo el uso de metodologías participativas asociadas a la elaboración de vídeos por parte del alumno de manera autónoma ha favorecido su implicación hacia el trabajo, resultando una estrategia idónea para regular el aprendizaje en las actividades demandadas. Además, en el grupo que elaboró los vídeos existen diferencias significativas entre aquellos que no usan las redes sociales y aquellos que lo hacen diariamente, siendo estos últimos los que han percibido una mayor transferencia del aprendizaje.

Los resultados reflejan que antes de llevarse a cabo la asignatura no existían diferencias significativas entre los grupos en ninguno de los dos factores, obteniendo incluso valores similares. Este aspecto, en los diseños pretest-postest, es positivo ya que refleja con mayor rigurosidad los efectos que ha tenido el plan de intervención en las variables estudiadas (Brogan \& Kutner, 2012). Además los valores medios de cada factor no llegaban a los 3.5 puntos, lo que indica que la percepción de los estudiantes sobre la implicación en las tareas y la regulación del trabajo en asignaturas precedentes no era muy elevada. Este hecho justifica más si cabe el desarrollo de esta experiencia, ya que se proponen una serie de procedimientos de actuación vinculados al uso de instrumentos concretos que favorecen la regulación y organización del trabajo, atendiendo a las pautas metodológicas utilizadas en cada grupo. Como indican Hortigüela, Pérez-Pueyo and Salicetti (2015) los docentes deben reflexionar sobre la percepción que tiene el alumnado sobre la metodología utilizada en el aula, ya que es la única manera de ajustar las pretensiones de aprendizaje entre docente y discente. 
Cuando la asignatura finaliza las valoraciones cambian mucho, obteniéndose una diferencia significativa en el factor de regulación del trabajo en el grupo que utilizó los vídeos respecto al comienzo. Parece por lo tanto que la experiencia permitió al alumnado planificar su trabajo respecto a la búsqueda de información, presentación del vídeo, delimitación de roles en su exposición y debate posterior conjunto para la extracción de conclusiones significativas, siendo esto último fundamental para autoevaluar el aprendizaje adquirido (Rodríguez, Ibarra, \& Jiménez-Vergara, 2013). Como consecuencia de ello en el postest, y en relación a este factor, las diferencias son significativas entre grupos, habiendo más de uno punto de diferencia. Parece por lo tanto que el alumno valora positivamente el hecho de que se le permita profundizar en su trabajo de manera autónoma, facilitándole el uso de instrumentos que le sirvan para ser miembro activo de su propio aprendizaje. Esto además permite la indagación sobre nuevas vías que favorezcan compartir experiencias tanto con compañeros de clase como con profesionales que trabajen en red, algo que enriquece más si cabe la experiencia (Roith, 2013). Por lo tanto, y a pesar de que las medias de los dos factores de estudio sean considerablemente más altas en el grupo A, en el que no utilizó los vídeos como elemento formativo también se incrementaron los valores, aunque ligeramente. Esto indica que con la metodología más tradicional utilizada en el grupo B los alumnos no experimentaron un descenso en la implicación hacia las tareas, lo que refleja que las experiencias prácticas utilizadas en las sesiones no desmotivan al alumno hacia la asignatura. En este sentido, Redelius and Hay (2012) reflejan trabajos en los que se muestra una correlación positiva entre la valoración que realiza el alumno sobre la asignatura y la calificación obtenida, lo que no muestra una veracidad adecuada en las respuestas.

Respecto a la percepción del alumnado sobre la aplicabilidad del aprendizaje obtenido, el único grupo en el que se obtienen diferencias significativas en función de las variables independientes de estudio es el que utilizó los vídeos como instrumento formativo. La variable es la referente a la asiduidad en el uso de las redes sociales fuera del aula. Son los alumnos que lo hacen diariamente los que consideran que la experiencia de la creación de los vídeos es aplicable a otros contextos, mientras que los que hacen periódicamente, menos. Experiencias como Carmona Morales, Jimenez-Hornero, Vazquez and Morilla (2012) indican que no siempre está relacionado el manejo virtual que tengan los alumnos fuera del aula con herramientas concretas de trabajo desarrolladas en clase. Por ello parece importante diferenciar entre el tiempo útil de aprendizaje invertido en medios virtuales y aquel que no está regulado y delimitado para tal fin. En el grupo B, que no se han utilizado medios virtuales, no se encuentran diferencias en ninguna de las variables. Esto puede ser producido a la menor discrepancia de opiniones entre estudiantes en cada uno de los dos factores, quizás por estar más acostumbrados a cursar asignaturas con este tipo de procedimientos. Esto puede observarse en los valores más bajos de las 
DT que muestra este grupo en el postest. En la edad y el conocimiento previo de la herramienta del Hangout no se encuentran diferencias intragrupales, lo que contrasta con otros estudios (Dappolone, 2013) que indican que los alumnos más jóvenes tienen una mejor percepción sobre su uso. Se manifiesta también la buena acogida de la herramienta de google utilizada, lo que indica su sencillez en su manejo.

Los datos obtenidos en las entrevistas de los docentes ratifican la parte cuantitativa del estudio, reflejándose una disparidad evidente en el propio conocimiento de las TIC y su consecuente implementación en la asignatura. El docente del grupo A otorga una mayor relevancia a la importancia que tiene la metodología para que el alumno se implique en las tareas, destacando la delimitación de criterios aceptados por la clase como medio fundamental para regular el trabajo. Esto conlleva al planteamiento de procedimientos que generen una autoevaluación y reflexión crítica sobre el aprendizaje generado, facilitando además de este modo el feedback entre iguales (Hortigüela, Pérez-Pueyo \& Abella, 2015). La docente del grupo B, pese a no trabajar las TIC, reconoce las posibilidades que pueden conllevar para el aprendizaje. En este sentido, el profesor que utilizó los vídeos manifiesta que la clave se encuentra no tanto en la enseñanza de multitud de instrumentos como en el uso real y aplicado de herramientas que den sentido a lo trabajado en el aula

\section{CONCLUSIONES}

En relación al primer objetivo, se ha comprobado cómo el grupo que elaboró de manera autónoma los vídeos virtuales como elemento formativo experimentó un aumento tanto en la implicación en las tareas como en la regulación del trabajo, siendo este factor el que experimentó una evolución significativa tanto en relación al pretest como entre grupos.

Respecto al segundo objetivo, en la variable independiente de asiduidad en el uso de las redes sociales se encontraron diferencias en relación a la aplicabilidad del aprendizaje generado. Esto sucedió en el grupo A, lo que indica una vivencia más positiva de la experiencia desarrollada en los estudiantes que tiene un mayor manejo de las TIC.

En respuesta al tercer objetivo, los dos docentes entrevistados manifiestan una disparidad de opiniones evidente en cuanto a la implantación de herramientas virtuales en el aula, a pesar de que la profesora del grupo B reconozca su posible utilidad para el aprendizaje. Esto se asocia al uso de una metodología más definida y rigurosa por parte del docente del grupo A.

Consideramos que el presente estudio puede ser de interés para todos aquellos docentes interesados en desarrollar propuestas metodológicas y evaluativas que garanticen la implicación del alumno en las tareas, utilizando para ello herramientas virtuales de fácil manejo y aplicación en el aula. El estudio presenta algunas limitaciones. En primer lugar únicamente se aplica la propuesta en una asignatura, 
por lo que sería interesante contrastar los datos con otras de diferentes titulaciones. Por otro lado, sería conveniente valorar la carga de trabajo para docente y alumnado que puede conllevar experiencias como esta, valorando de este modo más criterios para su utilización en clase

\section{REFERENCIAS}

\section{Publicaciones Periódicas:}

Armstrong, D. (2011). Students' Perceptions of Online Learning and Instructional Tools: A Qualitative Study of Undergraduate Students Use of Online Tools. Turkish Online Journal of Educational Technology, 10(3), 222-226.

Beckett, C., \& Clegg, S. (2007). Qualitative Data from a Postal Questionnaire: Questioning the Presumption of the Value of Presence. International Journal of Social Research Methodology, 10(4), 307-317. doi: 10.1080/13645570701401214

Blanco, A. (2013). El uso y el conocimiento de los contenidos de educación física en Internet del alumnado de segundo ciclo de Educación Secundaria Obligatoria. Estudio de caso. Enseñanza \& Teaching: Revista interuniversitaria de didáctica, 31(2), 69-92.

Bryman, A., Becker, S., \& Sempik, J. (2008). Quality Criteria for Quantitative, Qualitative and Mixed Methods Research: A View from Social Policy. International Journal of Social Research Methodology, 11(4), 261-276.

Castejón, F.J., Santos, M., y Palacios, A. (2013) Cuestionarios sobre metodología y evaluación en formación inicial en Educación Física. Revista Internacional de Medicina y Ciencias de la Actividad Física y el Deporte, 12(1), 1-23.

Cifuentes, P., \& Meseguer, P. (2015). Trabajo en equipo frente a trabajo individual. Ventajas del aprendizaje cooperativo en el aula de traducción. Tonos digital: Revista electrónica de estudios filológicos, 28, 1-21.

Craig, C., \& Friehs, C. (2013). Video and HTML: Testing Online Tutorial Formats with Biology Students. Journal of Web Librarianship, 7(3), 292-304.

Dappolone, M. (2013). Making Best Practices Better. Educational Leadership, 70(6), 69-72.

Del Valle, P., Morales, M., \& Sumano, A. (2011). Motivación y Autorregulación propiciadas mediante el uso del portafolio electrónico en los alumnos de nivel superior. Revista Iberoamericana de educación, 55, 173- 187.

Fugard, A., \& Potts, H. (2015). Supporting thinking on sample sizes for thematicanalyses: a quantitative tool. International Journal of Social Research Methodology, 22, 1-15. doi: 10.1080/13645579.2015.1005453

Hortigüela, D., Pérez Pueyo, A. y Abella, V. (2015). Perspectiva del alumnado sobre la evaluación tradicional y la evaluación formativa. Contraste de grupos en las mismas asignaturas. REICE. Revista Iberoamericana sobre Calidad, Eficacia y Cambio en Educación, 13(1), 35-48. Recuperado de

http://www.rinace.net/reice/numeros/arts/vol13num1/art3.pdf. ISSN: 1696-4713.

Flores, O. (2012). Tic y docencia universitaria ¿cambian las metodologías docentes según el grado de presencialidad de las asignaturas? El caso de la Universidad de Lleida. Pixel-Bit: Revista de medios y educación, 41, 63-76. 
Kenny, J., \& Fluck, A. (2014). The Effectiveness of Academic Workload Models in an Institution: A Staff Perspective. Journal of Higher Education Policy and Management, 36(6), 585-602.

Liu, H., Liu, J., \& Chi, X. (2014). Regulatory Mechanism of Self-Determination Involvement in Higher Education: Assessing Chinese Students' Experiences. Higher Education: The International Journal of Higher Education and Educational Planning, 67(1), 51-70.

Marín, V. (2014). Una propuesta de integración metodológica del Campus Virtual y herramientas web 2.0 en la asignatura. Responsabilidad contractual y extracontractual de tercer curso de los estudios de grado de Derecho. Revista de educación y derecho, 10, 1-19.

Martínez-Berruezo, M.A., \& García-Varela, A.B. (2013). Análisis de la influencia de la virtualización en la motivación del alumnado universitario de primer curso de Magisterio. Revista de Educación, 362, 42-68.

Rodríguez, G., Ibarra, M., \& Jiménez-Vergara, E. (2013). Autoevaluación, evaluación entre iguales y coevaluación: conceptualización y práctica en las universidades españolas. Revista de investigación en educación, 2(11), 198-210.

Roith, C. (2013). Las nuevas tecnologías de la información y comunicación en la planificación y realización de un curso virtual de máster universitario. Caracciolos: revista digital de investigación en docencia, 1, 1-11.

Schatz, E. (2012). Rationale and procedures for nesting semi-structured interviews in surveys or censuses. Population Studies: A Journal of Demography, 66(2), 183-195. doi: 10.1080/00324728.2012.658851

Vargas-Vargas, M., Mondejar-Jimenez, J., Santamaria, M.L., Alfaro-Navarro, J.L., \& Fernandez-Aviles, G. (2011). Cooperative Learning in Virtual Environments: The Jigsaw Method in Statistical Courses. Journal of International Education Research, 7(5), 1-8.

\section{Capítulo de un libro o entrada de un libro de consulta:}

Hall, J., \& Ryan, K. (2011). Educational Accountability: A Qualitatively Driven MixedMethods Approach. Qualitative Inquiry, 17(1), 105-115.

Hortigüela, D., Pérez Pueyo, A. y Salicetti, A. (2015). ¿Cómo percibe el alumnado universitario de educación física la evaluación recibida? Contraste de dos metodologías diferentes. Retos. Nuevas tendencias en Educación Física, Deporte y Recreación, 28, 66-71.

Gamlem, S., Smith, K. (2013). Student Perceptions of Classroom Feedback. Assessment in Education: Principles, Policy \& Practice, 20(2), 150-169.

Lau, K., \& Lee, P. (2015). The Use of Virtual Reality for Creating Unusual Environmental Stimulation to Motivate Students to Explore Creative Ideas. Interactive Learning Environments, 23(1), 3-18.

Smith, J. A. y Osborne, M. (2003). Interpretative Phenomenological Analysis. In J.A. Smith (Ed.) Qualitative psychology: A practical guide to research methods. (2nd ed., pp. 53-79). London: Sage. 
Redelius, K., \& Hay, P. (2012). Student Views on Criterion-Referenced Assessment and Grading in Swedish Physical Education. Physical Education and Sport Pedagogy, 17(2), 211-225.

\section{Libros completes:}

Brogan, D., \& Kutner, M. (2012). Comparative Analyses of Pretest-Posttest Research Designs. The American Statistician, 34(4), 229-232. doi: 10.1080/00031305.1980.10483034

Bulman, C., Lathlea, J., \& Gobbi, M. (2014). The Process of Teaching and Learning about Reflection: Research Insights from Professional Nurse Education. Studies in Higher Education, 39(7), 1219-1236.

Carmona, D., Jimenez-Hornero, J. E., Vazquez, F., Morilla, F. (2012). Educational Tool for Optimal Controller Tuning Using Evolutionary Strategies. IEEE Transactions on Education, 55(1), 48-57.

Corbetta, P. (2007). Metodologías y técnicas de investigación social. Madrid: McGraw Hill.

Denzin, N. K., \& Lincoln, Y. S. (1994). Handbook of Qualitative Research. Thousand Oaks, CA: Sage.

Saldaña, J. (2009). The Coding Manual for Qualitative Researchers. Thousand Oaks, CA: Sage.

\section{AUTOR/ES:}

\section{David Hortigüela Alcalá}

Doctor Internacional en Educación. Máster en Educación y Sociedades Inclusivas. Licenciado en Ciencias de la Actividad Física y del Deporte. Maestro Especialista en Educación Física. Graduado en Primaria mención en inglés. Posgrado en Bilingüismo. Docente en el Área de Expresión Corporal del Departamento de Didácticas Específicas. Autor de diversidad de artículos publicados en revistas científicas, libros, capítulos de libros y ponente en variedad de congresos nacionales e internacionales. Colaborador en varios proyectos $\mathrm{I}+\mathrm{D}+\mathrm{I}$ y de innovación docente. Revisor de diferentes revistas en el ámbito científico. Especialista en líneas de investigación vinculadas a la evaluación formativa, metodología y didáctica de la educación física, innovación en el aula y ciclos de investigación-acción.

\section{Ángel Pérez Pueyo}

Doctor en Ciencias de la Actividad y del Deporte. Maestro Especialista en Educación Física. Docente en el Área de Educación Física y Deportiva del CAFD de León. Autor de diversidad de artículos publicados en revistas científicas, libros, capítulos de libros y ponente en variedad de congresos nacionales e internacionales. Colaborador en varios proyectos I+D+I y de innovación docente. Revisor de diferentes revistas en el ámbito científico. Especialista en líneas de investigación vinculadas a la programación, trabajo de las competencias clave, aplicación de metodologías activas y participativas en el aula y de métodos cualitativos aplicados en el marco de investigación. 